\title{
Review Article \\ Far-Field Focus and Dispersionless Anticrossing Bands in Two-Dimensional Photonic Crystals
}

\author{
Xiaoshuang Chen, Renlong Zhou, Yong Zeng, Hongbo Chen, and Wei Lu \\ National Laboratory for Infrared Physics, Shanghai Institute of Technical Physics, Chinese Academy of Sciences, \\ Shanghai 200083, China
}

Received 7 November 2006; Revised 14 February 2007; Accepted 20 August 2007

Recommended by Ralf B. Bergmann

\begin{abstract}
We review the simulation work for the far-field focus and dispersionless anticrossing bands in two-dimensional (2D) photonic crystals. In a two-dimensional photonic-crystal-based concave lens, the far-field focus of a plane wave is given by the distance between the focusing point and the lens. Strong and good-quality far-field focusing of a transmitted wave, explicitly following the well-known wave-beam negative refraction law, can be achieved. The spatial frequency information of the Bloch mode in multiple Brillouin zones (BZs) is investigated in order to indicate the wave propagation in two different regions. When considering the photonic transmission in a 2D photonic crystal composed of a negative phase-velocity medium (NPVM), it is shown that the dispersionless anticrossing bands are generated by the couplings among the localized surface polaritons of the NPVM rods. The photonic band structures of the NPVM photonic crystals are characterized by a topographical continuous dispersion relationship accompanied by many anticrossing bands.
\end{abstract}

Copyright (c) 2007 Xiaoshuang Chen et al. This is an open access article distributed under the Creative Commons Attribution License, which permits unrestricted use, distribution, and reproduction in any medium, provided the original work is properly cited.

\section{INTRODUCTION}

As was shown by Vesselago over 30 years ago [1], left-handed materials (LHMs) possess a number of unusual electromagnetic effects including negative refraction and allow for a considerable control over light propagation. Years later, the LHM has attracted some theoretical and experimental attentions [2-27]. The phase velocity of the light wave propagating inside the medium points to the opposite direction of the energy flow. Thus, the Poynting vector and wave vector are antiparallel. Consequently, the light is refracted negatively. As a result, for such frequency ranges, the photonic crystal (PC) behaves as if the index of refraction is negative. The far-field focusing of acoustic waves has been shown in twodimensional sonic crystals consisting of hexagonal arrays of steel cylinders in air [28]. In further research, negative refraction allows for the focusing of a far-field radiation by concave rather than convex surfaces [29-31], with the advantage of reduced aberration for the same radius of curvature, and changes many commonly accepted aspects of conventional optical systems. One prominent example towards far-field focusing has been experimentally realized in a planoconcave lens by Vodo and Parimi et al. [31]. The possible applications of such a phenomenon to photonic devices are anticipated.
On the other hand, early research was largely concentrated on photonic band gap (PBG) materials consisting of positive and frequency-independent dielectrics. In the past few years, negative phase-velocity (NPV) materials [32-34], also named left-handed materials and negative refractive index media, have been successfully fabricated $[35,36]$. Recently, a periodic arrangement of NPV materials, namely, NPV-medium photonic crystals, has become the object of intense experimental and theoretical interest [37-41]. Like polaritonic PCs whose $\mu$ is homogeneous while $\varepsilon$ is frequencydependent $[42,43]$, the application of NPV materials in PCs is expected to introduce a whole range of exciting physical phenomena. For instance, a multilayer structure with stacking alternating layers of ordinary (positive- $n$ ) and NPV materials can result in an omnidirectional zero- $\bar{n}$ gap $[38,39]$, where the defect modes depend weakly on the incident angles [44]. In general, the permittivity and permeability of an NPV material are frequency-dependent [37]. In a split-ring resonator used to create negative magnetic materials, the permeability is normally expressed as $\mu(\omega)=1-\omega_{m} / \omega(\omega+i \gamma)$, where $\omega_{\mathrm{m}}$ is the effective magnetic plasma frequency, $\omega$ is the frequency of the light, and $\gamma$ represents the light absorption [37]. Due to these dispersive properties of NPV materials, the study of high-dimensional NPV-medium PCs thus becomes 


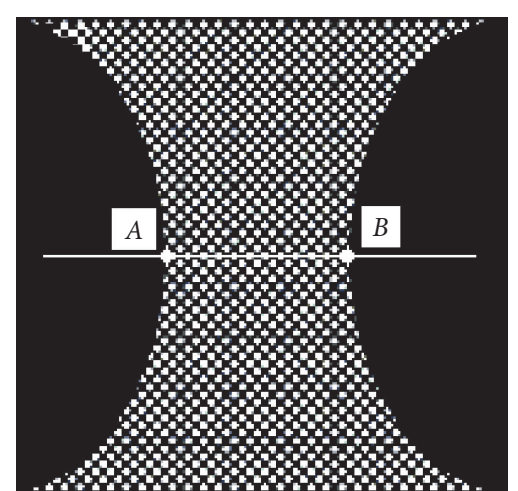

(a)

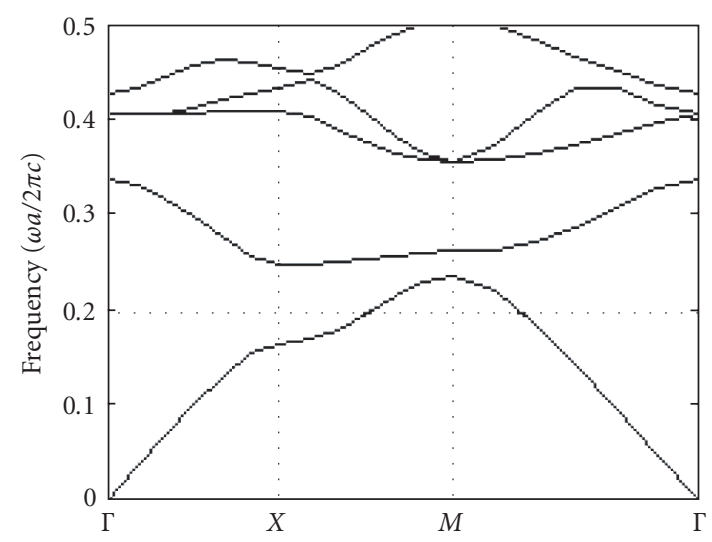

(b)

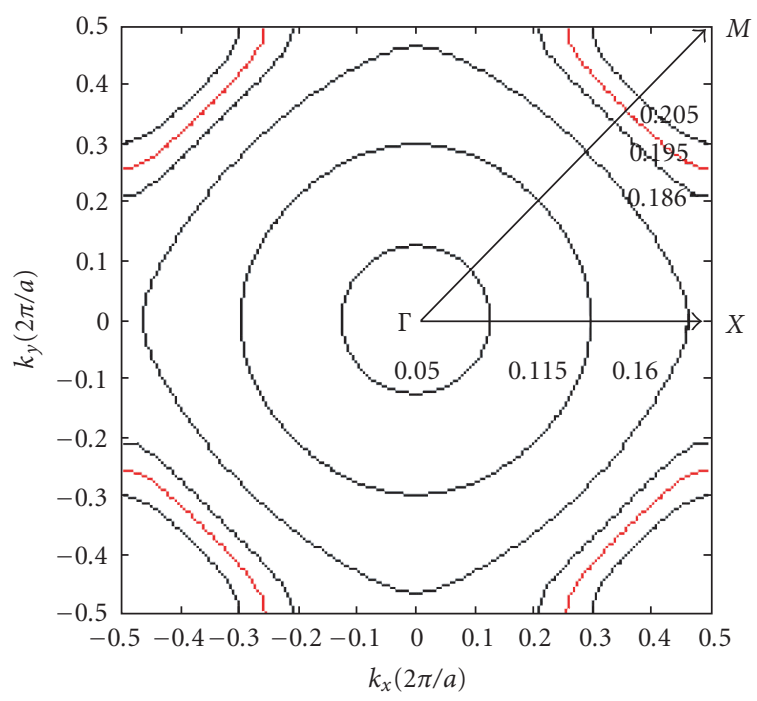

(c)

Figure 1: (a) The photonic-crystal-based concave lens; (b) band structure of the PC calculated with the plane wave expansion method; (c) the EFCs of the first band for the TE polarization mode. Frequency values are in units of $2 \pi c / a$. rather complicated for many conventional theoretical methods [45].

In this paper, we show that a $2 \mathrm{D}$ photonic-crystal-based concave lens achieves a strong far-field focusing effect. The incident waves are from the background material, propagating through a section of a PC designed for negative refraction at the considered frequency range, and exiting to the background material. The focusing of a plane wave is successfully observed. The spatial frequencies information of the Bloch mode is shown, describing the wave propagation. When considering negative phase-velocity medium, the optical properties are studied with the 2D PC in a square lattice of NPVmaterial cylindric rods embedded in air background. The whole PC structure is divided into thin slices along the wave propagation direction. It is assumed that each slice is surrounded by two thin air films with zero thickness. The EM fields in the air films and in each slice are expressed by the Floquet harmonics and the eigenmodes of the slice, respectively. Since the independent variable in these calculations is the frequency rather than the wave vector, it can therefore effectively simulate dispersive PCs, even though their permeability and permittivity are frequency-dependent and negative.

\section{FOCUSING PROPERTIES IN THE 2D PHOTONIC-CRYSTAL-BASED CONCAVE LENS}

The system we examine is a $2 \mathrm{D}$ photonic-crystal-based concave lens consisting of a square lattice of circular air holes (with lattice constant $a$ and hole radius $r=0.35 a$ ) in dielectric background with $\varepsilon=12.0$ as shown in Figure 1(a). The optical principal axis of the lens is normal along the $\Gamma M$ direction with the length $\mathrm{AB}=16.9 a$, and the curvature of the lens is 0.7092 . Here, the point $A(B)$ is located at the left (right) surface along the optical principal axis. Only $T E$ modes (magnetic field parallel to the axis of the holes) are considered.

To visualize and analyze the negative refractive effect and the far-field focusing, by using a plane-wave expansion method [12-14, 46], we have calculated the photonic band structure in Figure 1(b) and the equifrequency-surface contours (EFCs) in Figure 1(c) for TE mode in $\vec{k}$ space whose gradient vectors give the group velocities of the photonic modes. In Figure 1(c), the EFCs for five relevant frequencies are demonstrated, respectively. Note that throughout this paper the frequency is always in units of $2 \pi c / a$. The 0.05 and 0.115 contours are very close to a perfect circle, and therefore the group velocity at any point of the contour is collimated with the $\vec{k}$ vector and $\vec{k} \cdot \partial \omega / \partial \vec{k} \geq 0$, indicating that the crystal behaves like an effective homogeneous medium with positive effective index for the 0.05 and 0.115 frequencies. The contour with frequency 0.16 is a bit distorted from a circle, with the distance $|\vec{k}|$ along the $\Gamma M$ direction being slightly shorter than along the $\Gamma X$ direction, indicating that the crystal behaves like a positive effective index medium. In most parts of the 0.195 contour, the curve is quite flat in the middle of this contour and the surface is normal pointing to the $\Gamma M$ direction. In small parts of the 0.195 contour near the 
BZ edges, the surface is convex with respect to the $M$ point. For the 0.195 contour, the group velocity of the excited Bloch wave mode near the BZ edges is pointed towards the $M$ point, corresponding to an apparent negative refraction direction. The group velocity centered round the $\Gamma M$ direction in most parts of the 0.195 contour would point dominantly along the $\Gamma M$ direction.

\section{FOCUSING PROPERTIES OF A PLANE WAVE}

For photonic-crystal-based thin lab lens, only the near-field focusing is easily observed. In the photonic-crystal-based concave lens, we can find that the far-field focusing is formed. We perform a simulation for the incident plane wave at frequency $\omega=0.195(2 \pi c / a)$. A typical result of the intensity distribution is shown in Figure 2(a). The strong focusing of the waves is successfully observed. The surface of the field distribution for a plane wave incident to the PC surface is also shown in Figure 2(b). One can find easily that a far-field focusing of the plane wave is formed out of the PC concave lens, and the focusing keeps on in a steady state. The focal point is centered at $x=65 a$ and $y=0$; that is, the focal length is $65 a$ and it is very far from the right interface of the concave lens. The strong focal point has a width maximum of $4.5 a$. The intensity of the focusing is three times stronger than that of incident plane wave. The results confirm that far-field focusing is realizable and opens the door for the applications of the LHM in the far-field region.

Next, in order to discuss in more detail the focusing effect of plane wave source, the transversal ( $Y$ direction) light intensities at five positions, $x / a=-8.45,-4.23,0,4.23,8.45$, are shown in Figure 2(c), respectively. It is found that the intensity distribution in $x / a=-8.45$ is symmetric with respect to $x=0$. The intensity distribution along $Y$ shows how the EM wave transmits through the PC concave lens. In the left and right surface regions, we can clearly see that light intensity is in good agreement with the wave-beam negative refraction law. The wave with the frequency 0.195 propagating inside the PC concave lens is along the $\Gamma M$ direction. The focusing behavior is in complete agreement with the EFCs analysis.

Furthermore, at a given frequency 0.195 inside the PC concave lens, the propagation behavior can be explained by means of the spatial Fourier spectrum. In the Fourier transform, the wave vector $k_{x}$ is defined as the spatial frequency. For one particular $y$, a spatial Fourier transform of the field data presented in Figure 2(a) along the $x$ direction, around the focus region and inside the PC concave lens region, yields the spectrum $F\left(k_{x}\right)$ of the spatial frequency $k_{x}$ [15$17,47,48]$. Spatial frequency $k_{x}$ is in units of $2 \pi /(\sqrt{2} a)$. To improve the signal-to-noise ratio of the numerical simulation, the spectra $F\left(k_{x}\right)$ are summed for all $y$. We can find that the Bloch mode, which consists of more than one spatial frequency, is visualized in Figure 3. These spatial frequencies are separated by an integral number of $\pi /(\sqrt{2} a)$ along the $\Gamma M$ direction for the square lattice, as seen in Figure 3 for the first five zones. At frequency 0.195 , the intensity of the Bloch harmonics for the same Bloch mode inside the PC region with $k_{x}=0.705$ and 1.295 can be given as peaks (dotted line)

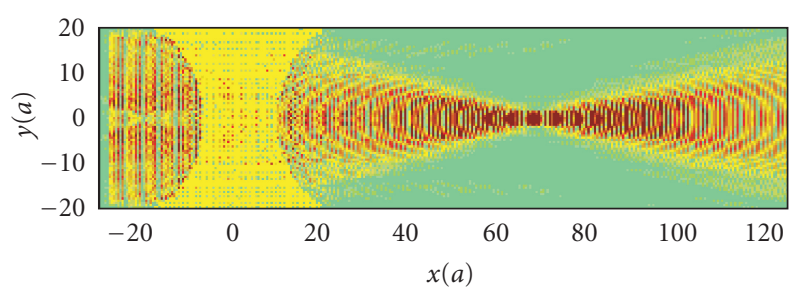

(a)

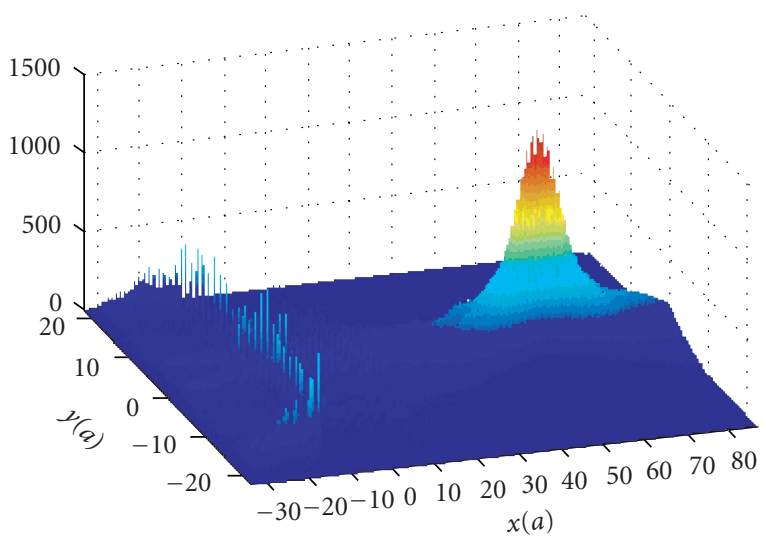

(b)

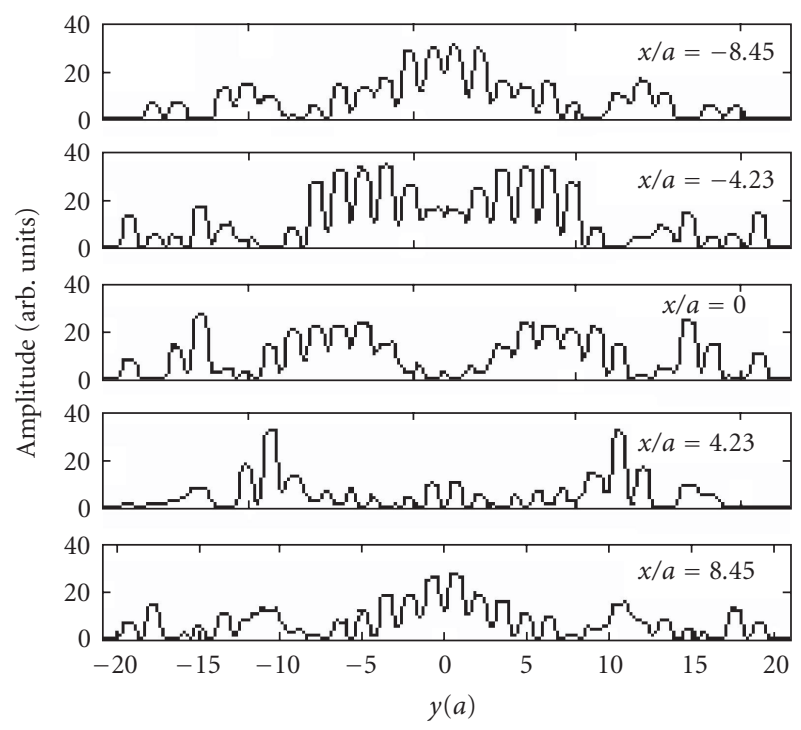

(c)

Figure 2: (a) The intensity distribution and (b) the surface of the field distribution for a plane wave incident to the PC interface. A high-quality far-field focus is formed out of the concave lens. (c) Intensity distributions along the transverse direction at five positions.

in Figure 3. One can find that the amplitudes of Bloch harmonics separated by $\pi /(\sqrt{2} a)$ are different. The exact physical interpretation behind this observation has been shown in $[47,48]$. As shown in Figure 3, near the focus region, only the spatial frequency of the Bloch mode $k_{x}=k_{0}=0.195 \sqrt{12}$ in units of $2 \pi /(\sqrt{2} a)$ ( $k_{0}$ is the wave number at frequency 0.195 


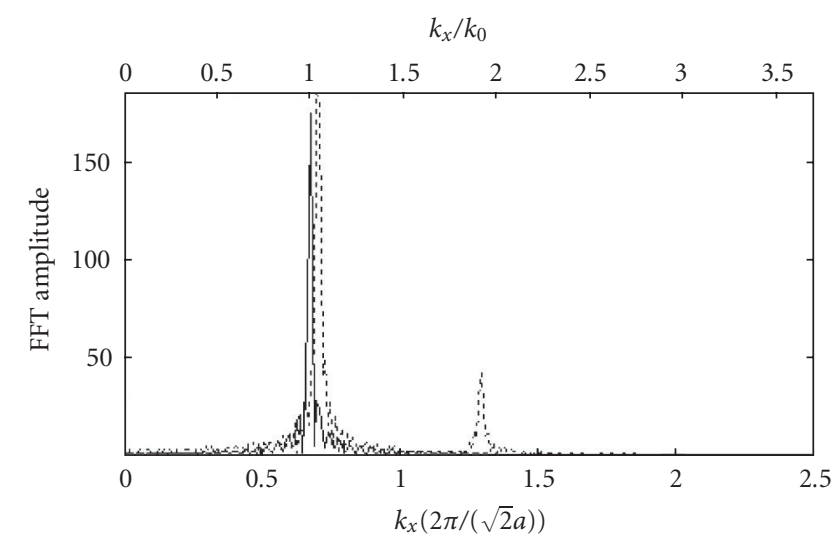

FIGURE 3: Spatial Fourier spectrum $F\left(k_{x}\right)$ versus spatial frequency $k_{x}$ for frequency 0.195 is summed around the focus region (solid line) and inside the PC concave lens region (dotted line), corresponding to the field data presented in Figure 2(a).

in dielectric background) with frequency 0.195 contributes to the propagation of EM wave. Therefore, when the waves leave the PC concave lens, the focusing of the waves is formed $[49,50]$.

\section{THE DISPERSIONLESS ANTICROSSING BANDS IN 2D PC WITH NEGATIVE PHASE-VELOCITY MEDIUM}

Based on the transfer-matrix approach, the photonic band structures and the corresponding spectra of $2 \mathrm{D} \mathrm{NPV-}$ material PCs are calculated. The 2D PC considered here is a square lattice of NPV-material cylindric rods embedded in air background. The rods are aligned along the $x$ axis and positioned periodically in the yz plane. The physical background of our method is as follows. (1) Divide the whole PC structure into thin slices along the $z$-axis direction; within each slice, $\epsilon$ and $\mu$ are approximated as $z$-independent (they are however $y$-dependent). (2) It is assumed that each slice is surrounded by two thin air films with zero thickness. (3) Express the EM field in the air films by Floquet harmonics. (4) Express the EM field in each slice by the eigenmodes in the slice. (5) Use the boundary conditions between EM fields in the slice and two neighboring air films to obtain the transfer matrix or the scattering matrix. (6) Use the obtained matrix to calculate the transmission spectrum of the PC. The photonic band structure of the PC can thus be obtained by using Bloch's theorem. For the sake of easy comparison, the relative permittivity $\varepsilon(\omega)$ and relative permeability $\mu(\omega)$ of the rods are adopted from [51]. The free-electron representation of $\varepsilon(\omega)$ is assumed:

$$
\varepsilon(\omega)=1-\frac{\omega_{p}^{2}}{\omega^{2}},
$$

where $\omega_{p}$ is the frequency of bulk longitudinal electron excitations, and

$$
\mu(\omega)=1-\frac{F \omega^{2}}{\omega^{2}-\omega_{0}^{2}} .
$$

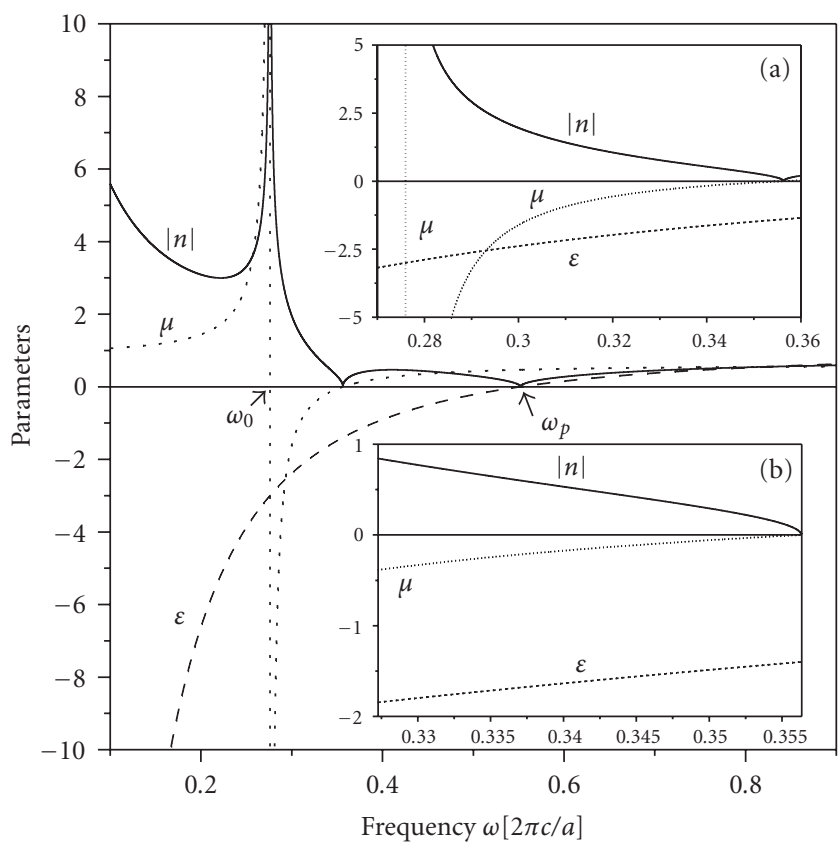

FIGURE 4: The permittivity $\varepsilon(\omega)$, permeability, and absolute refractive index $|n(\omega)|$ of the NPV-material cylindric rods as functions of $\omega$.

The parameters assumed here are $F=0.4, \omega_{p}=$ $0.552(2 \pi c / a), \omega_{0}=0.276(2 \pi c / a)$, and the radius of the rod $r=0.3 a$, where $a$ is the lattice constant and $c$ is the speed of light in vacuum. Notice that $\omega_{0}$ and $\omega_{p}$ used here are $1 / 20$ of those used in [51]. Really, we use the similar parameters of the left-hand rod as those in [51]. In [51], the unit is normalized with the radius of the rod, while in our paper, the parameters are normalized with the lattice constant $a$. Thus, the ratios of $\omega_{0}$ and $\omega_{p}$ to those of [51] are not their absolute values. Dissipative properties of NPV materials are neglected in (1) and (2), which is valid if the light absorption is very small. Moreover, the small light absorption has a very limited effect on the band structure.

Figure 4 shows $\mu(\omega), \varepsilon(\omega)$, and $|n(\omega)|$ of the NPVmaterial cylindric rods as functions of $\omega$. The exact positions of $\omega_{0}$ and $\omega_{p}$ are also shown in Figure 4. For the clarity of the figure, we plot $|n|$ instead of $n$ in the figure. We think it can make the figure look better if we use $|n|$ instead of $n$ because of the positive or negative refractive $n$-index. The refractive index $n$ values change the sign very quickly so that the value magnitude cannot be separated for the figure without the absolute values. For frequencies $\omega<\omega_{0}, \varepsilon(\omega)<0$ and $\mu(\omega)>0$, that is, the metamaterial is singly negative; for $\omega_{0}<\omega<0.35635(2 \pi c / a)$, both $\mu(\omega)$ and $\varepsilon(\omega)$ are negative so that the metamaterial is left-handed; for $0.35635(2 \pi c / a)<$ $\omega<\omega_{p}, \varepsilon(\omega)<0$ and $\mu(\omega)>0$, and the metamaterial is again singly negative; for $\omega>\omega_{p}$, both $\mu(\omega)$ and $\varepsilon(\omega)$ are positive, and the metamaterial is right-handed. Notice that $0.35635=\omega_{b} / 20$ which is used in [51], where $\omega_{b}$ is defined as $\omega_{b}=\omega_{0} / \sqrt{1-F}$.

The transmission spectrum along the same direction for a 16-layer structure is also calculated and shown in 


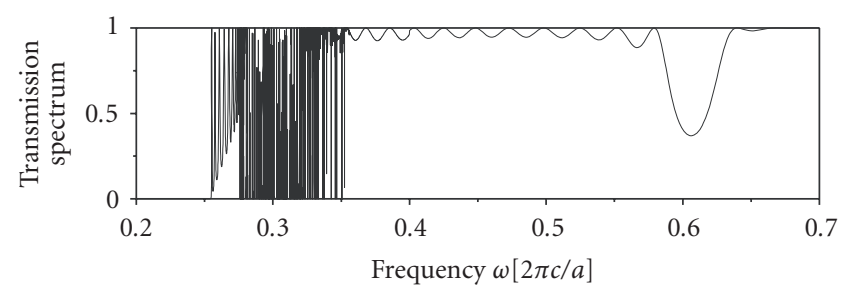

(a)

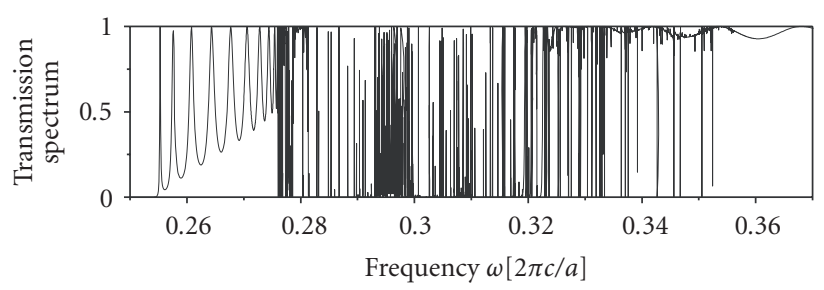

(b)

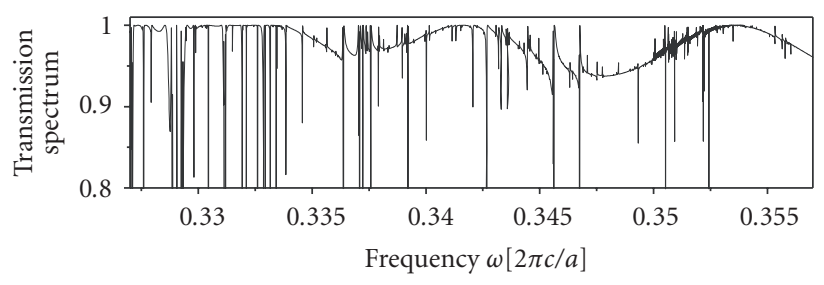

(c)

Figure 5: Transmission spectrum of the two-dimensional PC. (a) The frequency range in units of $2 \pi c / a$ is $[0.2,0.7]$. (b) The frequency range is $[0.25,0.37]$. (3) The frequency range is $[0.327$, 0.357 . Notice that more accurate results can be obtained by decreasing the increment frequency.

Figure 5(a). Two profound similarities between our periodic structure and the single rod are observed by comparing Figure 5(a) with the scattering resonances of a single NPVmaterial cylindric rod shown in [52, Figure 2(b)].

(1) Anticrossing bands appear when the metamaterial is NPV (see the inset of Figure 6 as an example), which are separated by very narrow band gaps and are almost dispersionless around the band edges. The group velocity is determined by the slope of the corresponding band curve. In such case of dispersionless band, the slope is very close to zero, so is the group velocity. Thus, the dispersionless bands (in other words, their group velocities are almost zero) lead to sharp resonant peaks in the transmission spectrum. Similar results have been reported in the scattering resonances of the single NPV-material rod [32], where resonances also exist in the NPV frequency region. We interpret this observation by collective couplings among highly localized resonance modes and surface polaritons of the NPV-material rods [51, 52]. It is noticed that the anticrossing bands also appear in polaritonic PCs [42].

(2) A narrow band gap exists slightly above $0.6(2 \pi c / a)$, corresponding to the valley in the transmission spectrum of Figure 5(a). A similar dip is also found around the same place in the scattering resonances of a single rod [51]. This is a di-

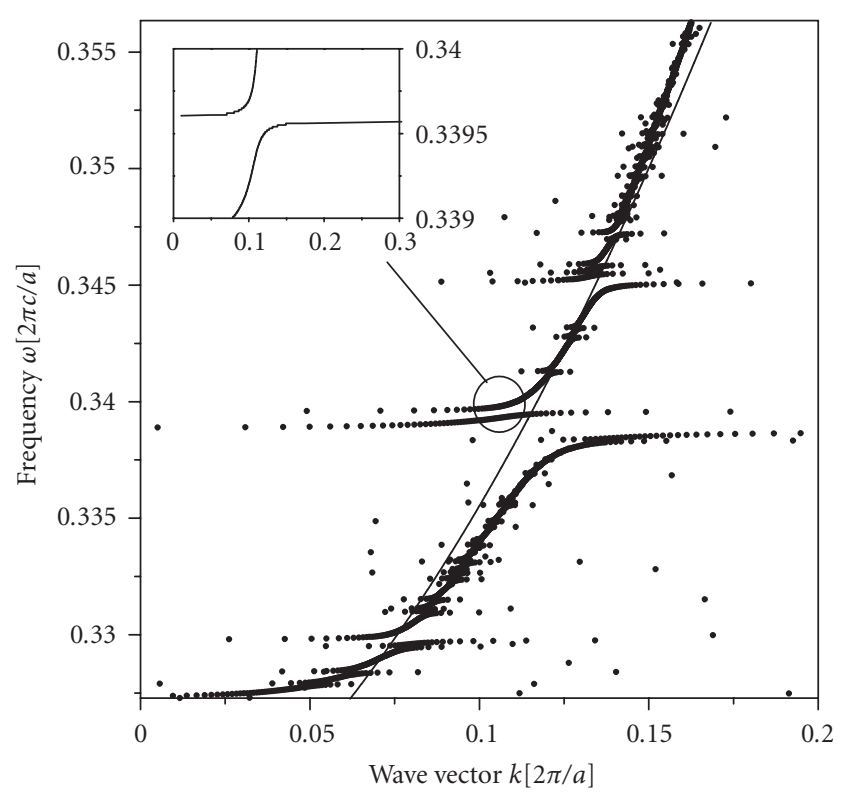

FIgure 6: The dispersion curves of the two-dimensional negative phase-velocity medium PC (dotted) and the corresponding effective structure (solid line). The inset shows the anticrossing bands around 0.340 .

rect result of the fact that the refractive index of the cylindric rod around $0.6(2 \pi c / a)$ is 0.275 , and that the volumeaveraged refractive index of the whole structure, roughly given by

$$
\bar{n}(\omega)=\frac{\left[\pi r^{2} \sqrt{\varepsilon(\omega) \mu(\omega)}+\left(a^{2}-\pi r^{2}\right) \times 1.0\right]}{a^{2}},
$$

becomes only 0.8 . This value is very close to the refractive index of vacuum so that the transmission rates around this narrow band gap are nonzero. They are about 37\%, as shown in Figure 5(a).

From these two similarities, it can be concluded that, as in a polaritonic $\mathrm{PC}$, the band structure of a high-dimensional NPV-material PC is governed principally by the surface polaritons localized in individual rods, whose frequencies are determined by the rods' geometries. The periodicity of the rods in the PC introduces only weak dispersion [42].

From $0.32728(2 \pi c / a)$ to $0.35635(2 \pi c / a)$, where the metamaterials are NVP, the dispersion relation can be topographically described as continual, accompanied by anticrossing bands. The dispersion relation here has the same physical origin as that in $\left[0.35635(2 \pi c / a), \omega_{p}\right]$, where the metamaterial is singly negative with a negative $\varepsilon(\omega)$ and a positive $\mu(\omega)$. This phenomenon can also be explained by the long-wavelength approximation; that is, when the wavelength of the propagating EM wave is much longer than the lattice constant of the $\mathrm{PC}$, the dispersion relationship becomes approximately linear, and the PC is effectively homogeneous. Notice that the refractive index of the NPV material in this region is very close to zero, as shown in Figure 4(b). In order to explain it more clearly, we plot the dispersion relationship of the effectively homogeneous structure in 


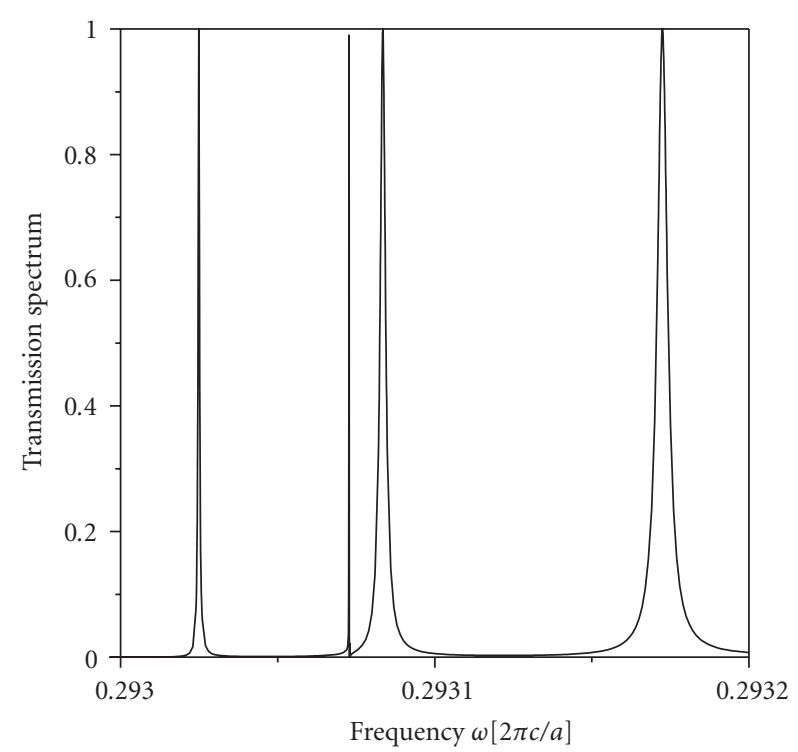

Figure 7: Transmission spectrum of the two-dimensional PC around $\omega a /(2 \pi c)=0.293125$.

Figure 6. More explicitly, the effective refractive index of the whole structure used here can be approximated by

$$
\bar{n}(\omega)=\frac{\left[-\pi r^{2} \sqrt{\varepsilon(\omega) \mu(\omega)}+\left(a^{2}-\pi r^{2}\right) \times 1.0\right]}{a^{2}},
$$

and the corresponding dispersion relationship, the thick solid line in Figure 6, is obtained from $k=\bar{n}(\omega) \omega / c$. Notice that the solid line is shifted upward by $0.0258(2 \pi c / a)$ in order to demonstrate that the two curves have similar slopes; that is, there are almost identical speeds of the energy propagation (given by $d \omega / d k$ ) in the effective structure and in the periodic structure.

When the volume-averaged refractive index of a multilayer structure $\bar{n}=\int_{0}^{a} n(x) d x / a / a$ equals zero with $a$ being the lattice constant, a band gap, namely, a zero- $\bar{n}$ gap, will appear in the band structure of the one-dimensional structure for normal incidence [38]. Our 2D sample also has a similar zero- $\bar{n}$ gap around $\omega a / 2 \pi c=0.293125$, as shown in Figure 7. When the refractive index of the cylindric rods around this frequency is about -2.5368 , the averaged refractive index of the $2 \mathrm{D}$ PC is zero by (4). In the 2D PC case, the zero- $\bar{n}$ gap is only one of many minigaps induced by the anticrossing interaction. It is very important to point out that the NPV material annihilates the effect of the surrounding normal material; a more accurate description of the effect of an NPV material is given in $[33,53]$. Moreover, a very sharp peak with very narrow bandwidth is shown at $0.29307275(2 \pi c / a)$ in Figure 7, and the ratio between the full width at half maximum and the peak frequency is extremely small (only $6.8 \times 10^{-8}(2 \pi c / a)$ ).

\section{CONCLUSION}

We have reviewed the numerical work on the strong farfield focusing and the dispersionless anticrossing bands in $2 \mathrm{D}$ photonic crystals. The far-field focus of a plane wave is obtained out of the concave lens. In comparison with the results of Vodo and Parimi, some similar focusing effects are shown, such as the far-field focusing of a plane wave [31]. The farfield focus of a plane wave is shown by the distance between the focusing point and the lens [19]. Moreover, the spatial frequencies information of the Bloch mode in multiple Brillouin zones is investigated, demonstrating the wave propagation in the 2D PC concave lens system. On the other hand, from the detailed comparisons between the periodic structure and a single NPVM cylindric rod, it has been shown that due to the coupling among the Anticrossing surface polaritons localized in neighboring NPVM rods, many anticrossing bands exist in the NPVM periodic structure. Furthermore, a topographical continual dispersion relation is found in a part of the negative phase-velocity frequency region, accompanied by many anticrossing bands, which has been explained by the long-wavelength approximation. Focusing and novel band structures are expected to be a significant step towards novel imaging optics and can lead to considerable changes in optical system design [54].

\section{ACKNOWLEDGMENTS}

This work is supported in part by the Chinese National Key Basic Research Special Fund (2007CB613206), Chinese National Science Foundation (60476040, 60576068), and Grand Foundation of Shanghai Science and Technology (05DJ14003), in addition to the computational support from Shanghai Supercomputer Center.

\section{REFERENCES}

[1] V. G. Veselago, "The electrodynamics of substances with simultaneously negative values of $\in$ and $\mu$," Soviet Physics Uspekhi, vol. 10, no. 4, pp. 509-514, 1968.

[2] R. A. Shelby, D. R. Smith, and S. Schultz, "Experimental verification of a negative index of refraction," Science, vol. 292, no. 5514 , pp. $77-79,2001$.

[3] D. R. Smith, D. Schurig, J. J. Mock, P. Kolinko, and P. Rye, "Partial focusing of radiation by a slab of indefinite media," Applied Physics Letters, vol. 84, no. 13, pp. 2244-2246, 2004.

[4] C. G. Parazzoli, R. B. Greegor, K. Li, B. E. C. Koltenbah, and M. Tanielian, "Experimental verification and simulation of negative index of refraction using Snell's law," Physical Review Letters, vol. 90, no. 10, Article ID 107401, 4 pages, 2003.

[5] J. B. Pendry, "Negative refraction makes a perfect lens," Physical Review Letters, vol. 85, no. 18, pp. 3966-3969, 2000.

[6] A. A. Houck, J. B. Brock, and I. L. Chuang, "Experimental observations of a left-handed material that obeys Snell's law," Physical Review Letters, vol. 90, no. 13, Article ID 137401, 4 pages, 2003.

[7] S. A. Ramakrishna and J. B. Pendry, "Removal of absorption and increase in resolution in a near-field lens via optical gain," Physical Review B, vol. 67, no. 20, Article ID 201101, 4 pages, 2003.

[8] P. V. Parimi, W. T. Lu, P. Vodo, J. Sokoloff, J. S. Derov, and S. Sridhar, "Negative refraction and left-handed electromagnetism in microwave photonic crystals," Physical Review Letters, vol. 92, no. 12, Article ID 127401, 2004. 
[9] E. Cubukcu, K. Aydin, E. Ozbay, S. Foteinopoulou, and C. M. Soukoulis, "Electromagnetic waves: negative refraction by photonic crystals," Nature, vol. 423, no. 6940, pp. 604-605, 2003.

[10] A. Grbic and G. V. Eleftheriades, "Overcoming the diffraction limit with a planar left-handed transmission-line lens," Physical Review Letters, vol. 92, no. 11, Article ID 117403, 2004.

[11] P. V. Parimi, W. T. Lu, P. Vodo, and S. Sridhar, "Photonic crystals: imaging by flat lens using negative refraction," Nature, vol. 426, no. 6965 , p. 404, 2003.

[12] C. Luo, S. G. Johnson, J. D. Joannopoulos, and J. B. Pendry, "All-angle negative refraction without negative effective index," Physical Review B, vol. 65, no. 20, Article ID 201104, 4 pages, 2002.

[13] Z. Ruan, M. Qiu, S. Xiao, S. He, and L. Thylén, "Coupling between plane waves and Bloch waves in photonic crystals with negative refraction," Physical Review B, vol. 71, no. 4, Article ID 045111, 7 pages, 2005.

[14] K. Ren, Z.-Y. Li, X. B. Ren, S. Feng, B. Cheng, and D. Zhang, "Three-dimensional light focusing in inverse opal photonic crystals," Physical Review B, vol. 75, no. 11, Article ID 115108, 6 pages, 2007.

[15] S. He, Z. Ruan, L. Chen, and J. Shen, "Focusing properties of a photonic crystal slab with negative refraction," Physical Review $B$, vol. 70, no. 11, Article ID 115113, 10 pages, 2004.

[16] C. Luo, S. G. Johnson, J. D. Joannopoulos, and J. B. Pendry, "Subwavelength imaging in photonic crystals," Physical Review $B$, vol. 68, no. 4, Article ID 045115, 15 pages, 2003.

[17] S. Xiao, M. Qiu, Z. Ruan, and S. He, "Influence of the surface termination to the point imaging by a photonic crystal slab with negative refraction," Applied Physics Letters, vol. 85, no. 19, pp. 4269-4271, 2004.

[18] E. Cubukcu, K. Aydin, E. Ozbay, S. Foteinopolou, and C. M. Soukoulis, "Subwavelength resolution in a two-dimensional photonic-crystal-based superlens," Physical Review Letters, vol. 91, no. 20, Article ID 207401, 4 pages, 2003.

[19] K. J. Webb and M.-C. Yang, "Generation and control of optical vortices using left-handed materials," Physical Review E, vol. 74, no. 1, Article ID 016601, 4 pages, 2006.

[20] A. A. Houck, J. B. Brock, and I. L. Chuang, "Experimental observations of a left-handed material that obeys Snell's law," Physical Review Letters, vol. 90, no. 13, Article ID 137401, 4 pages, 2003.

[21] E. J. Reed, M. Soljačić, and J. D. Joannopoulos, "Reversed doppler effect in photonic crystals," Physical Review Letters, vol. 91, no. 13, Article ID 133901, 4 pages, 2003.

[22] Y. Zeng, X. Chen, and W. Lu, "Modified spontaneous emission from a two-dimensional photonic crystal," Physical Review E, vol. 70, no. 4 2, Article ID 047601, 3 pages, 2004.

[23] S. Foteinopoulou, E. N. Economou, and C. M. Soukoulis, "Refraction in media with a negative refractive index," Physical Review Letters, vol. 90, no. 10, Article ID 107402, 4 pages, 2003.

[24] C.-H. Kuo and Z. Ye, "Optical transmission of photonic crystal structures formed by dielectric cylinders: evidence for nonnegative refraction," Physical Review E, vol. 70, no. 5, Article ID 056608, 4 pages, 2004.

[25] Z.-Y. Li and L.-L. Lin, "Evaluation of lensing in photonic crystal slabs exhibiting negative refraction," Physical Review B, vol. 68, no. 24, Article ID 245110, 7 pages, 2003.

[26] X. Zhang, "Subwavelength far-field resolution in a square twodimensional photonic crystal," Physical Review E, vol. 71, Article ID 037601, 4 pages, 2005.

[27] X. Zhang, "Effect of interface and disorder on the far-field image in a two-dimensional photonic-crystal-based flat lens,"
Physical Review B, vol. 71, no. 16, Article ID 165116, 7 pages, 2005.

[28] L. Feng, X.-P. Liu, Y.-B. Chen, et al., "Negative refraction of acoustic waves in two-dimensional sonic crystals," Physical Review B, vol. 72, no. 3, Article ID 033108, 4 pages, 2005.

[29] J. B. Pendry and D. R. Smith, "Reversing light with negative refraction,” Physics Today, vol. 57, no. 6, pp. 37-43, 2004.

[30] A. Martínez and J. Martí, "Analysis of wave focusing inside a negative-index photonic-crystal slab," Optics Express, vol. 13, no. 8, pp. 2858-2868, 2005.

[31] P. Vodo, P. V. Parimi, W. T. Lu, and S. Sridhar, "Focusing by planoconcave lens using negative refraction," Applied Physics Letters, vol. 86, no. 20, Article ID 201108, 3 pages, 2005.

[32] J. B. Pendry, "Negative refraction makes a perfect lens," Physical Review Letters, vol. 85, no. 18, pp. 3966-3969, 2000.

[33] D. R. Smith, J. B. Pendry, and M. C. K. Wiltshire, "Metamaterials and negative refractive index," Science, vol. 305, no. 5685, pp. 788-792, 2004.

[34] A. Lai, T. Itoh, and C. Caloz, "Composite right/left-handed transmission line metamaterials," IEEE Microwave Magazine, vol. 5, no. 3, pp. 34-50, 2004.

[35] D. R. Smith, W. J. Padilla, D. C. Vier, S. C. Nemat-Nasser, and S. Schultz, "Composite medium with simultaneously negative permeability and permittivity," Physical Review Letters, vol. 84, no. 18 , pp. 4184-4187, 2000.

[36] R. A. Shelby, D. R. Smith, and S. Schultz, "Experimental verification of a negative index of refraction," Science, vol. 292, no. 5514, pp. 77-79, 2001.

[37] S. A. Ramakrishna, "Physics of negative refractive index materials," Reports on Progress in Physics, vol. 68, no. 2, pp. 449-521, 2005.

[38] J. Li, L. Zhou, C. T. Chan, and P. Sheng, "Photonic band gap from a stack of positive and negative index materials," Physical Review Letters, vol. 90, no. 8, Article ID 083901, 4 pages, 2003.

[39] I. V. Shadrivov, A. A. Sukhorukov, and Y. S. Kivshar, "Complete band gaps in one-dimensional left-handed periodic structures," Physical Review Letters, vol. 95, no. 19, Article ID 193903, 2005.

[40] L. Feng, X.-P. Liu, Y.-F. Tang, et al., "Tunable negative refraction in a two-dimensional active magneto-optical photonic crystal," Physical Review B, vol. 71, no. 19, Article ID 195106, 6 pages, 2005.

[41] L. Chen, S. He, and L. Shen, "Finite-size effects of a left-handed material slab on the image quality," Physical Review Letters, vol. 92, no. 10, Article ID 107404, 2004.

[42] K. C. Huang, P. Bienstman, J. D. Joannopoulos, K. A. Nelson, and S. Fan, "Phonon-polariton excitations in photonic crystals," Physical Review B, vol. 68, no. 7, Article ID 075209, 12 pages, 2003.

[43] Y. Zeng, X. Chen, and W. Lu, "Propagation loss in twodimensional polaritonic photonic crystal waveguides," Physics Letters A, vol. 351, no. 4-5, pp. 319-322, 2006.

[44] H. Jiang, H. Chen, H. Li, Y. Zhang, and S. Zhu, "Omnidirectional gap and defect mode of one-dimensional photonic crystals containing negative-index materials," Applied Physics Letters, vol. 83, no. 26, pp. 5386-5388, 2003.

[45] Y. Zeng, Y. Fu, X. Chen, W. Lu, and H. Ågren, “Optical properties of two-dimensional negative-phase-velocity-medium photonic crystals," Physical Review E, vol. 73, no. 6, Article ID 066625, 5 pages, 2006.

[46] K. M. Ho, C. T. Chan, and C. M. Soukoulis, "Existence of a photonic gap in periodic dielectric structures," Physical Review Letters, vol. 65, no. 25, pp. 3152-3155, 1990. 
[47] H. Gersen, T. J. Karle, R. J. P. Engelen, et al., "Direct observation of Bloch harmonics and negative phase velocity in photonic crystal waveguides," Physical Review Letters, vol. 94, no. 12, Article ID 123901, 2005.

[48] H. Gersen, T. J. Karle, R. J. P. Engelen, et al., "Real-space observation of ultraslow light in photonlc crystal waveguides," Physical Review Letters, vol. 94, no. 7, Article ID 073903, 2005.

[49] M. Qiu, L. Thylén, M. Swillo, and B. Jaskorzynska, "Wave propagation through a photonic crystal in a negative phase refractive-index region," IEEE Journal of Selected Topics in Quantum Electronics, vol. 9, no. 1, pp. 106-110, 2003.

[50] A. Berrier, M. Mulot, M. Swillo, et al., "Negative refraction at infrared wavelengths in a two-dimensional photonic crystal," Physical Review Letters, vol. 93, no. 7, Article ID 073902, 2004.

[51] S. Ancey, Y. Décanini, A. Folacci, and P. Gabrielli, "Surface polaritons on left-handed cylinders: a complex angular momentum analysis," Physical Review B, vol. 72, no. 8, Article ID 085458, 18 pages, 2005.

[52] R. Ruppin, "Extinction properties of a sphere with negative permittivity and permeability," Solid State Communications, vol. 116, no. 8, pp. 411-415, 2000.

[53] A. Lakhtakia, "On perfect lenses and nihility," International Journal of Infrared and Millimeter Waves, vol. 23, no. 3, pp. 339-343, 2002.

[54] P. Vodo, P. V. Parimi, W. T. Lu, S. Sridhar, and R. Wing, "Microwave photonic crystal with tailor-made negative refractive index," Applied Physics Letters, vol. 85, no. 10, pp. 1858-1860, 2004. 

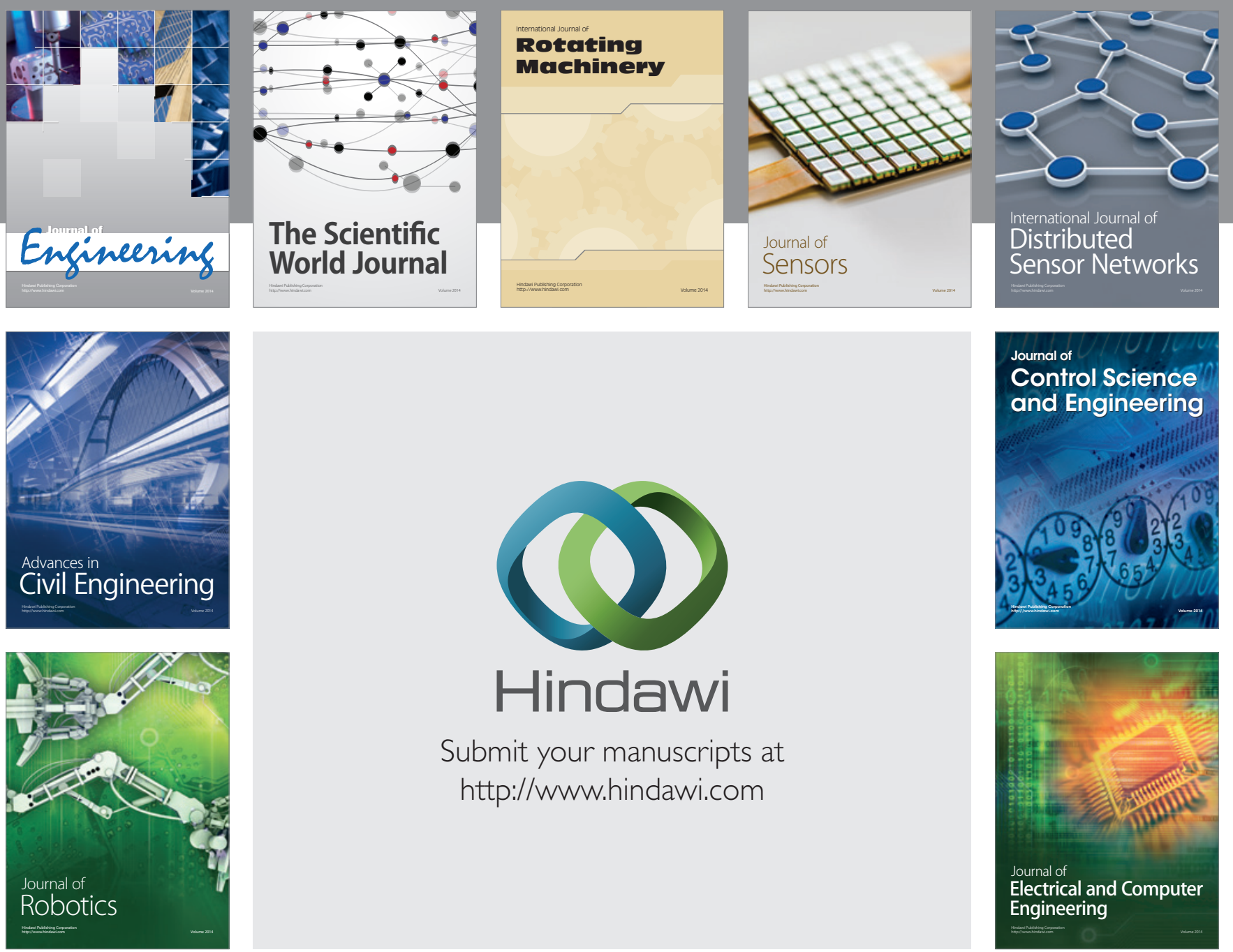

Submit your manuscripts at

http://www.hindawi.com
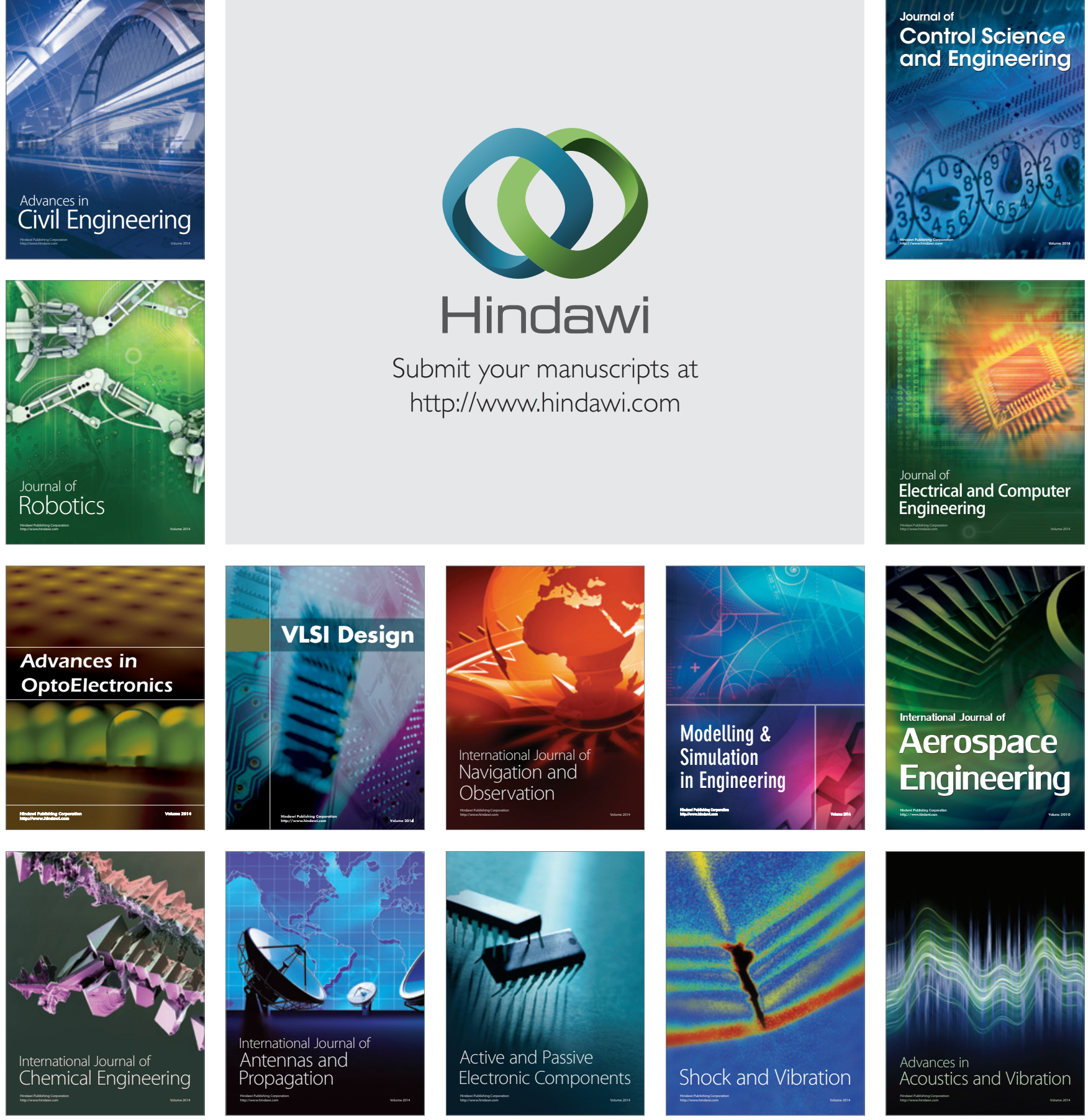